\title{
Psychological well-being in individuals with mild cognitive impairment
}

\author{
This article was published in the following Dove Press journal: \\ Clinical Interventions in Aging \\ 8 May 2014 \\ Number of times this article has been viewed
}

\author{
Nicola Gates ${ }^{1-3}$ \\ Michael Valenzuela ${ }^{3}$ \\ Perminder S Sachdev ${ }^{1,2,4}$ \\ Maria A Fiatarone Singh ${ }^{5,6}$ \\ 'School of Psychiatry, ${ }^{2}$ Centre for \\ Healthy Brain Ageing (CheBA), \\ University of New South Wales, \\ Sydney, NSW, Australia; ${ }^{3}$ Regenerative \\ Neuroscience Group, Brain and \\ Mind Research Institute, University \\ of Sydney, Sydney, NSW, Australia; \\ ${ }^{4}$ Neuropsychiatric Institute, Prince \\ of Wales Hospital, Sydney, NSW, \\ Australia; ${ }^{5}$ Exercise Health and \\ Performance Faculty Research Group, \\ Sydney Medical School, The University \\ of Sydney, Lidcombe, NSW, Australia; \\ ${ }^{6} \mathrm{Hebrew}$ SeniorLife, Boston, MA, and \\ Jean Mayer USDA Human Nutrition \\ Research Center on Aging at Tufts \\ University, Boston, MA, USA
}

Objectives: Cognitive impairments associated with aging and dementia are major sources of burden, deterioration in life quality, and reduced psychological well-being (PWB). Preventative measures to both reduce incident disease and improve PWB in those afflicted are increasingly targeting individuals with mild cognitive impairment (MCI) at early disease stage. However, there is very limited information regarding the relationships between early cognitive changes and memory concern, and life quality and PWB in adults with MCI; furthermore, PWB outcomes are too commonly overlooked in intervention trials. The purpose of this study was therefore to empirically test a theoretical model of PWB in MCI in order to inform clinical intervention.

Methods: Baseline data from a convenience sample of 100 community-dwelling adults diagnosed with MCI enrolled in the Study of Mental Activity and Regular Training (SMART) trial were collected. A series of regression analyses were performed to develop a reduced model, then hierarchical regression with the Baron Kenny test of mediation derived the final threetiered model of PWB.

Results: Significant predictors of PWB were subjective memory concern, cognitive function, evaluations of quality of life, and negative affect, with a final model explaining $61 \%$ of the variance of PWB in MCI.

Discussion: Our empirical findings support a theoretical tiered model of PWB in MCI and contribute to an understanding of the way in which early subtle cognitive deficits impact upon PWB. Multiple targets and entry points for clinical intervention were identified. These include improving the cognitive difficulties associated with MCI. Additionally, these highlight the importance of reducing memory concern, addressing low mood, and suggest that improving a person's quality of life may attenuate the negative effects of depression and anxiety on PWB in this cohort.

Keywords: positive aging, quality of life, memory concern

\section{Introduction}

Dementia is one of the principal causes of disability and decreased life quality among older adults. ${ }^{1}$ Coincident with a worldwide acceleration of dementia burden, there has been a sharp rise in quality of life (QoL) research in this field. ${ }^{2,3}$ Growing expectations for positive aging amongst older adults and policy concern about the rising costs of age-related health care and institutionalization underlie this trend. ${ }^{2}$ In fact, low life quality is a strong predictor of adverse health outcomes such as nursing home placement and death. ${ }^{4}$ Consequently, QoL outcomes are now recommended as essential in dementia prevention and intervention research. ${ }^{5}$

Despite the universal recognition that QoL is important, no single consensus definition of QoL is available, as definitions vary by theoretical and disciplinary perspectives. ${ }^{1-3,6}$
Correspondence: Nicola Gates School of Psychiatry, University of New South Wales, Randwick, Sydney, NSW 203I, Australia

Tel +6I 29969330 I

Fax +6I 293823774

Email nicolagates@bigpond.com 
A related but distinct concept that is viewed as a marker of successful aging is psychological well-being (PWB)., Recent definitions of PWB focus on eudaimonic well-being, which incorporates psychological concepts such as mastery, social connectedness, and self-acceptance. ${ }^{9}$ Additionally, research indicates that older adults emphasize $\mathrm{PWB}$, rather than biomedical factors, as they rate well-being as a priority despite the presence of disease and disability. ${ }^{8}$ In contrast, QoL often refers to hedonic concepts such as satisfaction with different domains of life, including health, finances, and recreation. ${ }^{10}$ Despite these differences, $\mathrm{PWB}$ is often not explicitly examined or is subsumed into the generic concept of QoL. ${ }^{11-17}$ The terms and constructs PWB and QoL are also frequently applied in research without definition ${ }^{15,18,19}$ with many studies confusing the terms and mixing outcome measures or simply avoiding defining terms. ${ }^{15,18}$

Maintaining life quality is highly relevant for those with neurodegenerative disorders as there is no effective cure. ${ }^{20}$ Jonker et al provided a three-tiered hierarchical model of QoL with PWB as the ultimate focus ${ }^{17}$ to improve treatment outcomes in dementia.

However, for the purposes of dementia prevention, interventions are increasingly targeting those in the early preclinical stage of the disease, ${ }^{21}$ often diagnosed with mild cognitive impairment (MCI). ${ }^{22}$ However, the vast majority of studies have focused upon dementia ${ }^{20}$ and no model of PWB has been developed for MCI. Additionally, clinical trials that enroll people with MCI have generally not examined QoL and PWB as outcome variables, with a recent review of cognitive interventions in MCI indicating that only two of 14 trials had QoL outcomes. ${ }^{23}$ Therefore, based upon
Jonker's 2004 model for Alzheimer's disease, we present a theoretical hierarchical model of PWB in MCI, shown in Figure 1, in order to inform intervention. This theoretical model includes both objective measures of disease and subjective measures of PWB and QoL, consistent with recent recommendations. ${ }^{24}$

Level 1 "clinical aspects of disease" (Jonker's term) $)^{17}$ are operationalized as the diagnostic criteria for MCI: subjective memory concern; mild cognitive impairment assessed on objective cognitive measures; and intact activities of daily living. ${ }^{22}$ "Clinical aspects not related to dementia" (Jonker's term $)^{17}$ includes covariates age, education, sex, and negative affect. External environment is defined here as social network, which has been found to influence health and life quality. ${ }^{25}$

Level 2 "evaluation of each domain" (Jonker's term) $)^{17}$ is operationalized as self-ratings of satisfaction across different aspects of life including health. Level 3 PWB, as defined above, is similar to the concepts of "positive productive aging" or "successful aging", 8,26 and is the ultimate clinical outcome.

It was originally postulated that level 1 factors would be interrelated and have discrete links with level 2 QoL and level 3 PWB, and that changes in clinical aspects of disease would be reflected in changes in evaluations of PWB ${ }^{17}$ However, this model was never empirically tested and it has been argued that such evaluation of QoL and PWB is necessary to advance our understanding of the field. ${ }^{27}$ Therefore, the purpose of this study was to empirically test the Jonker et al 2004 model of PWB within a group of older adults diagnosed with MCI. Based upon our model, it was hypothesized that:

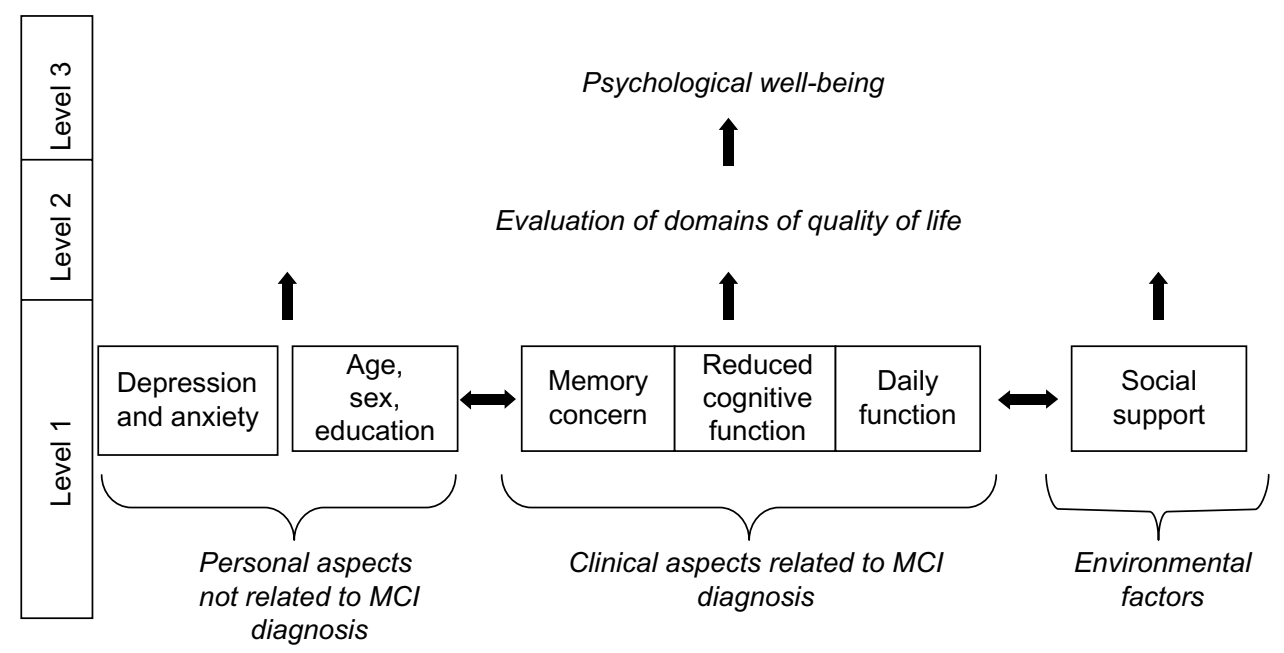

Figure I Hierarchical model of psychological well-being in mild cognitive impairment based upon Jonker's 2004 model of quality of life for dementia. Note: Jonker's terms in italics.

Abbreviation: $\mathrm{MCl}$, mild cognitive impairment. 
1. level 1 clinical aspects of MCI, negative affect, and social environment will be interrelated;

2. level 1 factors will be related to the level 2 evaluations of quality of life factors;

3. level 1 and level 2 factors will both be related to PWB; and, importantly;

4. level 2 factors will mediate the relationship between level 1 factors and PWB.

\section{Methods}

The data were drawn from the Study of Mental Activity and Regular Training (SMART) trial published by Gates et al ${ }^{28}$ and for which ethical approval was obtained from the Sydney South West Area Health Service (HREC Ref.08/RPAH/106), University of Sydney Human Research Ethics (HREC: 06-2008/11094), University of New South Wales (HREC: 08152), and signed informed consent was obtained from all participants (ANZCTR 83075).

\section{Participants}

Participants $(\mathrm{N}=100)$ were enrolled in the Sydney SMART trial $^{28}$ and were community-dwelling adults aged over 55 years with diagnosis of MCI: self-reported memory complaint; objective cognitive deficit based on a minimental status examination (MMSE) ${ }^{31}$ score of 23-29; and no dementia (Clinical Dementia Rating of 0.5 or below). ${ }^{32}$ Primary exclusion criteria of the SMART trial were clinical depression, unstable medical conditions, and other progressive neurological diseases. Full inclusion and exclusion details can be found in our published protocol. ${ }^{28}$

\section{Measures}

For details of the full neuropsychological battery and psychological test instruments, see the SMART protocol. ${ }^{28}$

Level 1 clinical aspects of MCI were the three common diagnostic criteria measured separately. Subjective memory concern can be validly assessed via memory complaint and a person's self-rated capacity to perform daily memory tasks, ${ }^{29}$ and both methods were used here. A study-specific questionnaire of seven items relating to severity of current memory complaints provided a memory complaint score (MCS), and self-rated memory function was assessed with the Memory Awareness Rating Scale-Memory Function Scale (MARS-MFS).$^{30}$ Cognitive function was measured with the MMSE ${ }^{31}$ the Alzheimer's Disease Assessment Scale-Cognitive Subscale (ADAS-Cog) ${ }^{32}$, and multidomain neuropsychological measures. These measures were: Trail Making Test (TMT);33 Symbol Digit Modalities Test
(SDMT) $;{ }^{33}$ Logical Memory I and II subtests of the Wechsler Memory Scale 3rd Edition; ${ }^{34}$ the ADAS-Cog three memory recall trials with a total score of correctly-recalled words for the assessment of list learning; Benton Visual Retention Test-Revised 5th Edition; ${ }^{35}$ controlled oral word association (COWAT); Category fluency (animal naming) $;^{33}$ and the Matrices and Similarities subtests of the Wechsler Adult Intelligence Scale 3rd Edition. ${ }^{36}$ Daily function was measured on the Bayer-Activities of Daily Living (B-ADL) ${ }^{37}$ as a self-rating scale of capacity to perform instrumental activities of daily life.

Level 1 nonclinical MCI factors were environment and negative affect. Negative affect was measured with the Geriatric Depression Scale (GDS) 15-items scale, ${ }^{38,39}$ and with the Depression Anxiety and Stress Scale 21(DASS).$^{40}$ Satisfaction with social environment was assessed on the eleven items of the abbreviated Duke Social Support Index $(\mathrm{DSSI})^{41}$ providing a satisfaction score regarding the size and structure of respondents, social network.

Level 2 evaluations of QoL involved measuring hedonic aspects of QoL obtained from the 15-item Quality of Life Scales (QOLS), ${ }^{42}$ the SF-36v2 ${ }^{\text {TM }},{ }^{43}$ and the Life Satisfaction Scale (LSS). ${ }^{12}$ The QOLS measures level of satisfaction across five domains of life: material and physical well-being; relationships with other people; social, community, and civic activities; personal development and fulfillment; and recreation. ${ }^{42}$ The Scale of Psychological Well-Being (SPWB) and the QOLS have been differentiated as measuring two distinct constructs. ${ }^{10}$ The SF-36v2 ${ }^{\mathrm{TM}}$ is a clinician-administered scale on which respondents rate eight areas: physical functioning; role functioning; bodily pain; general health; vitality; social functioning; role-emotional functioning; and mental health. The scoring algorithm generates two summary scores; a physical component score (SF-36 PCS) and mental component score (SF-36 MCS). The LSS is a global validated single item seven-point delighted-terrible rating scale. ${ }^{6}$

Level 3 psychological well-being concerned with eudaimonic factors measured with the 84-item SPWB ${ }^{13}$ across six domains - autonomy; environmental mastery; personal growth; positive relations with others; purpose in life; and self-acceptance - with respondents required to rate their level of agreement with each item.

\section{Procedures}

All results reported here were derived from baseline data collected before randomization. Sociodemographic and health status data were obtained through face-to-face interviews and assessment using structured interviews and self-report scales. 
One experienced neuropsychologist acquired all cognitive and psychological data.

\section{Statistical analysis}

Demographic details are reported as means and standard deviations (SD) for continuous variables and percentages for categorical variables. The distributions of all continuous variables used in the following analyses were examined and, if necessary, transformed to approximate the normal distribution more closely. Preliminary regressions were performed between PWB and all variables within the same level to avoid issues associated with colinearity. Relationships within level 1 variables (clinical aspects of MCI, social support, and negative affect) were analyzed using a series of regression analyses. For these analyses, each independent variable was entered separately into each of the regression models, together with control variables age, sex, education. Negative effect was entered as a control variable where appropriate to the particular analysis. Multivariate regression analyses were used to examine significant level 1 and level 2 predictors of SPWB and significant level 1 predictors of level 2 variables. Model reduction was carried out using the backwards elimination method with the $P$-value for item removal set at 0.10 . Regression using the stepwise procedure was performed to isolate the relative contribution of each level in the hierarchical model. Potential mediation of the effects of level 1 variables on SPWB by level 2 variables was examined using the method described by Baron and Kenny. ${ }^{44}$ All data analyses were performed using IBM SPSS version 21 (IBM Corporation, Armonk, NY, USA). A $P$-value of $<0.05$ was considered indicative of statistical significance.

\section{Results}

There are no missing values in any table as a full data set was acquired. Demographic information is shown in Table 1. The sample had a mean age of 70 years, was predominantly female $(68 \%)$, and all participants had completed secondary schooling (mean 13.4 years of schooling). Mild cognitive

Table I Baseline demographic characteristics of participants

\begin{tabular}{ll}
\hline & Mean (SD) range \\
\hline Age (years) & $70.1(6.6) 53.7-86.6$ \\
Sex (women) & $68 \%$ \\
Language background other than English & $15 \%$ \\
Education (years) & $13.4(3.6) 6-2$ I \\
Clinical Dementia Rating & $0.1(0.2) 0-0.5$ \\
Rated 0 & $71 \%$ \\
Rated 0.5 & $29 \%$ \\
Mini-mental State Exam score (0-30) & $27.5(1.5) 23-29$ \\
\hline
\end{tabular}

Note: $\mathrm{N}=100$.

Abbreviation: SD, standard deviation. impairment was evident (mean MMSE 27.47, SD 1.46: mean Clinical Dementia Rating 0.14, SD 0.22). Descriptive statistics for the measures of clinical aspects of MCI, negative affect, social support, evaluations of QoL, and PWB are presented in Table 2. Scores on the DASS-depression and DASS-anxiety scales were transformed with square root transformation due to nonnormality of the distributions prior to analyses. Memory complaints (mean 2.84, SD 1.37) and intact capacity to perform activities of daily living (mean

Table 2 Means (standard deviation) and score range (maximum score) for all measures

\begin{tabular}{|c|c|c|c|}
\hline Domain & Measure & Mean (SD) & Range (MS) \\
\hline PWB & SPWB & 249 (33) & $|65-32|(324)$ \\
\hline \multirow{4}{*}{$\begin{array}{l}\text { Evaluations of } \\
\text { quality of life }\end{array}$} & SF36 MCS & $52.5(6.6)$ & $30.3-52.5(100)$ \\
\hline & SF36 PCS & $48.7(8.7)$ & $23.2-61.2(100)$ \\
\hline & QOLS & $87(12)$ & $54-112(I \mid 2)$ \\
\hline & LSS & $3(1)$ & I-6 (7) \\
\hline \multicolumn{4}{|l|}{ environment } \\
\hline \multirow[t]{4}{*}{ Negative affect } & DASS depression & $2(3)$ & $0-13(21)^{\#}$ \\
\hline & DASS anxiety & $2(3)$ & $0-13(21)^{\#}$ \\
\hline & DASS stress & $4(3)$ & $0-15(21)^{\#}$ \\
\hline & GDS & $2(1)$ & $0-16(15)^{\#}$ \\
\hline \multirow[t]{2}{*}{ Daily function } & B-ADL & $0.1(0.1)$ & $0-0.2$ \\
\hline & & & $(>3 \text { excluded })^{\#}$ \\
\hline \multirow[t]{2}{*}{ Memory concern } & MCS & 2.84 (I.37) & $0.0-6.0(7)^{\#}$ \\
\hline & MARS-MFS & $38.4(5.7)$ & $22-55(56)$ \\
\hline Cognitive & ADAS-Cog & $7.9(3.4)$ & $2-18(70)^{\#}$ \\
\hline \multirow[t]{12}{*}{ function } & ADAS-Learning & $19.9(3.9)$ & $7-28(30)$ \\
\hline & Logical I & II.2 (3.9) & $3-20(25)$ \\
\hline & Logical II & $9.15(4.4)$ & $0-20(25)$ \\
\hline & BVRT & $6.1(1.7)$ & $1-10(10)$ \\
\hline & TMT B & $89.9(33.4)$ & $\begin{array}{l}38-242 \text { seconds } \\
(300)\end{array}$ \\
\hline & COWAT & $38.0(12.8)$ & $16-67$ \\
\hline & & & (no maximum) \\
\hline & Animal fluency & $12.0(4.9)$ & $\begin{array}{l}\text { 4-23 (no } \\
\text { maximum) }\end{array}$ \\
\hline & Matrices & $12.0(4.9)$ & $4-23(26)$ \\
\hline & Similarities & $19.7(5.1)$ & $6-30(33)$ \\
\hline & TMT A & $41.0(15.6)$ & $\begin{array}{l}20-117 \text { seconds } \\
(300)\end{array}$ \\
\hline & SDMT & $44.2(9.6)$ & $15-65(\mid 10)$ \\
\hline
\end{tabular}

Notes: "Higher score indicates worse function or symptoms. Matrices and Similarities are subtests of the Wechsler Adult Intelligence Scale 3 rd edition. Animals refers to category word fluency.

Abbreviations: ADAS-Cog, Alzheimer's Disease Assessment Scale-cognitive; ADAS-Learning, list learning trial of ADASC Cog; B-ADL, Bayer-Activities of Daily Life; BVRT, Benton Visual Retention Test revised 5th edition; COWAT, controlled oral word association test; DASS, Depression Anxiety and Stress Scale; DSSI, Duke Social Support Index; GDS, Geriatric Depression Scale; LSS, life satisfaction scale; Logical, immediate logical delayed logical memory subscale of Wechsler Memory Scale 3rd edition; MARS-MFS, Memory Awareness Rating Scale-Memory Functioning Scale; MCS, memory concern scale; MMSE, mini-mental state exam; MS, maximum score; PWB, psychological well-being; QOLS, quality of life scale; SD, standard deviation; SDMT, Symbol Digit Modality Test; SF36 PCS, SF-36v2 ${ }^{\text {TM }}$ physical component score; SF36 MCS, SF-36v2 ${ }^{\text {TM }}$ mental component score; SPWB, Scale of Psychological Wellbeing; TMT, Trail Making Test; TMT A, numbers; TMT B, numbers and letters. 
score 0.13 , SD 0.06) were evident, commensurate with diagnosis of MCI. The cohort had minimal levels of depression consistent with exclusion criteria and high levels of life satisfaction (LSS mean 2.7, SD 1.0), QoL (QOLS mean 87.1, SD 11.6), and PWB (SPWB mean 249.4, SD 33).

\section{The relationships between clinical aspects of $\mathrm{MCl}$ and other level I variables}

A series of regression analyses with the memory concern variables as dependent variables (DVs) on each cognitive variable and B-ADL as an independent variable (IV), and B-ADL (DV) on each cognitive variable (IV) are presented in Table S1. Demographics and negative affect variables were included in each analysis as control variables. Women reported significantly more dependency in activities of daily living (B-ADL; beta regression value $(\beta) 0.20, P<0.05$ ) compared to men while men had lower self-rated memory function (MARS-MFS; $\beta-0.24, P<0.05$ ) than women. Younger participants had greater memory complaints (MCS; $\beta-0.25, P<0.05$ ). More memory complaints (MCS) were significantly linked to greater cognitive difficulty (ADASCog; $\beta$ 0.21, $P<0.05$ ), worse memory (Logical I; $\beta-0.24$, $P<0.05$; Logical II; $\beta-0.22, P<0.05)$, and $(\beta-0.21$, $P<0.05)$. Higher self-rated memory function (MARSMFS) was significantly associated with better memory performance (Logic II; $\beta$ 0.20, $P<0.05$ ) and less cognitive difficulty (ADAS-Cog; $\beta-0.22, P<0.05$ ). Greater difficulties on B-ADL were significantly associated with higher memory complaint (MCS; $\beta 0.25, P<0.05$ ) and lower selfrated memory function (MARS-MFS; $\beta-0.35, P<0.05$ ), but not with objective cognitive function.

The results of regression analyses for the effects of clinical aspects of MCI on social support and negative affect variables are presented in Table S2. Each of the clinical aspects of MCI variables were entered singly into each regression equation, with age, sex, and education included in each analysis as control variables. Higher education was related to fewer DASS-depression symptoms $(\beta-0.22, P>0.05)$ and DASS-anxiety symptoms $(\beta-0.27, P<0.05)$, but age and sex were not related to negative affect scores.

Generally, both subjective and objective cognitive function and negative affect were inversely related, as anticipated. After controlling for demographics, increasing memory complaint (MCS) was significantly associated with more depressive symptoms on GDS ( $\beta 0.29, P<0.005)$. Better self-rated memory function (MARS-MFS) was significantly associated with fewer depressive symptoms (GDS; $\beta-0.29, P<0.005$; DASS-depression; $\beta-0.25$,
$P<0.05)$ and stress $(\beta-0.25, P<0.05)$. Worse ADAS-Cog performance was related to more depressive symptoms on GDS $(\beta 0.28 P<0.005)$ and greater stress (DASS-stress; $\beta 0.34, P<0.05)$. Higher delayed memory on Logical II was associated with lower depressive symptoms (GDS; $\beta-0.22, P<0.05)$ and better visual memory with lower stress (DASS-stress; $\beta-0.35, P<0.001$ ), depressive symptoms (DASS-depression; $\beta-0.28, P<0.05$ ), and anxiety symptoms (DASS-anxiety; $\beta-0.24, P<0.05$ ). Worse verbal fluency (COWAT) was associated with greater depressive and anxiety scores (DASS-depression; $\beta-0.36, P<0.05$; DASSanxiety; $\beta-0.29, P<0.05)$. Slower TMTA performance was significantly associated with more negative affect (DASSdepression; $\beta 0.33, P<0.05$; DASS-anxiety; $\beta 0.32, P<0.05$; DASS-stress; $\beta$ 0.21, $P<0.05)$. Faster visual-motor speed on Symbol Digit Modalities Test was significantly linked to lower depression (DASS-depression; $\beta-0.28, P<0.05$ ) and anxiety (DASS-anxiety $\beta-0.22, P<0.05$ ). By contrast, functional independence (B-ADL score) was not related to any negative affect measure.

Higher satisfaction with social support (DSSI) was associated with better self-reported memory function (MARSFCS; $\beta 0.21, P<0.05$ ), less cognitive difficulty (ADAS-Cog; $\beta-0.26, P<0.05$ ), and better verbal fluency (COWAT; $\beta 0.23$, $P<0.05)$.

\section{Hierarchical model: relationships between levels I, 2, and 3}

Regression of level 3 SPWB (DV) onto each single independent variable, and multivariate regression of SPWB on all level 1 variables (clinical aspects of MCI, mood, social support) and on both level 1 and level 2 evaluations of life quality variables combined, are presented in Table 3. In the reduced models, ratings of SPWB were not significantly associated with any demographic variable. The final set of significant predictors of SPWB, with both level 1 and 2 variables as IVs, were level 1 objective cognitive function, self-rated memory function and negative affect, and level 2 evaluations of QoL. Better SPWB was associated with lower verbal fluency (COWAT; $\beta-0.23, P<0.00$ ) but better matrices performances (Matrices; $\beta$ 0.22, $P<0.05$ ), cognitive level (MMSE; $\beta 0.19$, $P<0.05$ ), and self-rated memory function (MARS-FCS; $\beta 0.29, P<0.05)$, lower anxiety $(\beta-0.25, P<0.05)$ and depression (DASS-depression; $\beta-0.19, P<0.05$ ), and better evaluations of QoL (QOLS; $\beta$ 0.39, $P<0.05$ ). In multivariate analyses, higher SPWB was associated with lower physical component QoL (SF36 PCS; $\beta-0.23, P<0.05)$ and was unrelated to mental component (SF36 MCS; $\beta-0.12, P>0.1$ ) or 
Table 3 Regression analyses for the effects of level I variables, and the effects of level I and level 2 variables combined, on SPWB

\begin{tabular}{|c|c|c|c|c|c|}
\hline \multirow[t]{2}{*}{ Independent variables } & \multirow{2}{*}{$\begin{array}{l}\text { Univariate } \\
\text { analyses }\end{array}$} & \multicolumn{4}{|c|}{ Dependent variable level 3 SPWB } \\
\hline & & $\begin{array}{l}\text { Level I variables } \\
\text { as IVs }\end{array}$ & Reduced model $^{\mathrm{a}}$ & $\begin{array}{l}\text { Level I and } \\
\text { level } 2 \text { as IVs }\end{array}$ & $\begin{array}{l}\text { Reduced } \\
\text { model }^{\mathrm{a}}\end{array}$ \\
\hline \multicolumn{6}{|l|}{ Demographics } \\
\hline Age & $0.12(0.22)$ & $0.22(0.03)^{*}$ & & $0.12(0.18)$ & \\
\hline Sex & $-0.03(0.72)$ & $0.00(0.47)$ & & $0.04(0.65)$ & \\
\hline Education & $0.07(0.45)$ & $0.04(0.69)$ & & $0.03(0.81)$ & \\
\hline \multicolumn{6}{|l|}{ Negative affect } \\
\hline GDS & $-0.03(0.00)^{*}$ & $0.08(0.35)$ & & $0.05(0.53)$ & \\
\hline DASS anxiety & $-0.04(0.00)^{*}$ & $-0.21(0.04)^{*}$ & $-0.21(0.03)^{*}$ & $-0.28(0.00)^{*}$ & $-0.25(0.00)^{*}$ \\
\hline DASS depression & $-0.05(0.00)^{*}$ & $-0.29(0.0 \mathrm{I})^{*}$ & $-0.25(0.01)^{*}$ & $-0.29(0.00) *$ & $-0.19(0.03)^{*}$ \\
\hline DASS stress & $-0.04(0.00)^{*}$ & $0.11(0.320)$ & & $0.13(0.24)$ & \\
\hline \multicolumn{6}{|l|}{ Social support } \\
\hline DSSI & $0.37(0.00)^{*}$ & $0.21(0.01)^{*}$ & $0.23(0.00)^{*}$ & $0.05(0.55)$ & \\
\hline \multicolumn{6}{|l|}{ Memory concern } \\
\hline MCS & $-0.10(0.34)$ & $0.06(0.56)$ & & $0.12(0.23)$ & \\
\hline MARS-MFS & $0.44(0.00)^{*}$ & $0.32(0.00)^{*}$ & $0.35(0.00)^{*}$ & $0.32(0.00)^{*}$ & $0.29(0.00)^{*}$ \\
\hline \multicolumn{6}{|l|}{ Daily function } \\
\hline$B-A D L$ & $-0.16(0.19)$ & $0.07(0.42)$ & & $0.03(0.73)$ & \\
\hline \multicolumn{6}{|l|}{ Cognitive function } \\
\hline ADAS-learning & $0.04(0.66)$ & $-0.09(0.4 \mathrm{I})$ & & $-0.06(0.60)$ & \\
\hline ADAS-Cog & $0.31(0.00)^{*}$ & $0.20(0.15)$ & & $0.15(0.23)$ & \\
\hline MMSE & $0.19(0.05) *$ & $0.14(0.16)$ & $0.15(0.04)^{*}$ & $0.18(0.04)^{*}$ & $0.19(0.00)^{*}$ \\
\hline TMT A & $0.32(0.00)^{*}$ & $0.13(0.18)$ & & $0.11(0.22)$ & \\
\hline TMT B & $0.16(0.13)$ & $0.04(0.68)$ & & $0.01(0.88)$ & \\
\hline Matrices & $0.11(0.29)$ & $0.24(0.03)^{*}$ & $0.17(0.05)^{*}$ & $0.28(0.01)^{*}$ & $0.22(0.01)^{*}$ \\
\hline Similarities & $-0.02(0.79)$ & $0.16(0.19)$ & & $-0.03(0.7 \mathrm{I})$ & \\
\hline Animal fluency & $0.05(0.64)$ & $0.01(0.88)$ & & $-0.04(0.77)$ & \\
\hline COWAT & $0.03(0.77)$ & $-0.29(0.02)^{*}$ & $-0.22(0.0 \mathrm{I})^{*}$ & $-0.31(0.01)^{*}$ & $-0.23(0.00)^{*}$ \\
\hline Logical I & $-0.12(0.22)$ & $-0.22(0.25)$ & $-0.18(0.02)^{*}$ & $-0.16(0.30)$ & \\
\hline Logical II & $-0.03(0.73)$ & $0.07(0.70)$ & & $0.10(0.50)$ & \\
\hline BVRT & $0.35(0.00)^{*}$ & $-0.07(0.49)$ & & $0.00(0.98)$ & \\
\hline SDMT & $0.19(0.08)$ & $-0.105(0.38)$ & & $0.01(0.92)$ & \\
\hline \multicolumn{6}{|c|}{ Level 2 evaluations of quality of life } \\
\hline QOLS & $0.60(0.00)^{*}$ & - & - & $0.28(0.0 \mathrm{I})^{*}$ & $0.39(0.00)^{*}$ \\
\hline SF36 PCS & $0.02(0.8 I)$ & - & - & $-0.30(0.00)^{*}$ & $-0.23(0.00)^{*}$ \\
\hline SF36 MCS & $0.41(0.00) *$ & - & - & $-0.12(0.29)$ & \\
\hline LSS & $-0.36(0.00)^{*}$ & - & - & $-0.10(0.28)$ & \\
\hline Total $R^{2}$ & & $0.60, P<0.000 *$ & $0.54, P<0.000^{*}$ & $0.69, P<0.000^{*}$ & $\begin{array}{l}0.61 \\
P<0.000 *\end{array}$ \\
\hline
\end{tabular}

Notes: ${ }^{\text {TT }}$ The reduced regression models were obtained using the backwards elimination procedure. Standardized regression coefficients are reported (numbers in parentheses are the $P$-values); *significance level of 0.05 . DASS-depression, DASS-anxiety, and DASS-stress are subscales of the Depression Anxiety and Stress Scale 21 . Logical I and Logical II are, respectively, logical memory immediate and delayed memory subtests of Wechsler Memory Scale 3rd edition. Animal refers to category fluency with animal names. Matrices and Similarities are subtests of Wechsler Adult Intelligence Scale 3rd edition.

Abbreviations: ADAS-Cog, Alzheimer's Disease Assessment Scale-cognitive; ADAS-Learning, learning word lists across the memory trails of the ADAS-Cog; B-ADL, Bayer-Activities of Daily Living; BVRT, Benton Visual Retention Test-Revised 5th edition; COWAT, controlled oral word association; DASS, Depression Anxiety and Stress Scale 2I; DSSI, Duke Social Support Index; GDS, Geriatric Depression Scale; IVs, independent variables; LSS, life satisfaction scale; MARS-MFS, Memory Awareness Rating Scale-Memory Functioning Scale; MCS, memory complaint score; MMSE, mini-mental status examination; PWB, psychological well-being; QOLS, quality of life scales; SDMT, Symbol Digit Modalities Test; SF36 MCS, SF-36v2 ${ }^{\text {TM }}$ mental health component score; SF36 PCS, SF-36v2 ${ }^{\text {TM }}$ physical component score; SPWB, scale of PWB; TMT, Trail Making Test; TMT A, numbers; TMT B, numbers and letters.

LSS $(\beta-0.10, P<0.1)$. The final reduced regression model of SPWB, obtained using backwards elimination procedure, is shown in Table 3 .

Hierarchical regression analysis, using the variables in the reduced model shown in Table 3, was performed to determine the contribution of the clinical aspects of $\mathrm{MCI}$, after controlling for negative affect, and the contribution of the evaluation of QoL variables in addition to all other variables in the model. Depression and anxiety were entered first, followed by clinical aspects of MCI (memory function and cognitive function), and then evaluations of QoL at step 3. The full model explained more than half the variance of SPWB (total $R^{2}=0.61$; $P<0.001)$. Memory concern and cognitive function made an additional significant contribution to the explanation of variance in SPWB $\left(R^{2}\right.$ change $\left.=0.17 ; P<0.001\right)$ above 
the contribution of depression and anxiety. Evaluations of QOLS and SF36PCS were entered last and contributed an additional $R^{2}$ of $0.19(P<0.001)$, indicating that level 2 evaluations of QoL predict additional variance in SPWB above level 1. A depiction of the final regression model is shown in Table 4.

\section{Mediation}

Applying Baron and Kenny's criteria for mediation, ${ }^{44}$ only two level 2 variables (QOLS and SF36-PCS) had statistically significant effects on SPWB when all variables were entered (see Table 3), and could potentially be included in mediation analyses. Two separate linear regressions were conducted with QOLS and SF36-PCS as DVs and level 1 variables as IVs providing reduced models following backwards elimination shown in Table S3. Next, only those level 1 variables that had statistically significant effects on SPWB (without QOLS and SF36-PCS in the equation [Table 3]), and on QOLS and SF36-PCS (Table S3), satisfied Baron and Kenny's criteria for inclusion. Inspection of the results in Tables 3 and S3 indicates that DASS-anxiety and DASS-depression, social

Table 4 Hierarchical regression model of level I and level 2 variables on the Scale of Psychological Wellbeing (SPWB)

\begin{tabular}{lll}
\hline Theoretical model & $\begin{array}{l}\text { Independent } \\
\text { variables }\end{array}$ & $\begin{array}{l}\text { SPWB } \\
\text { dependent }\end{array}$ \\
\hline $\begin{array}{l}\text { Level I } \\
\text { Negative affect }\end{array}$ & $\begin{array}{l}\text { DASS depression } \\
\text { DASS anxiety }\end{array}$ & $\begin{array}{l}-0.19(0.03)^{*} \\
-0.25(0.00)^{*}\end{array}$ \\
& & $R^{2}$ change \\
& Cognitive function & $0.25(0.00)^{*}$ \\
Clinical aspects of $\mathrm{MCl}$ & MMSE & $0.19(0.02)^{*}$ \\
& Matrices & $0.22(0.00)^{*}$ \\
& COWAT & $-0.23(0.00)$ \\
& Memory concern & \\
& MARS-MFS & $0.27(0.00)^{*}$ \\
& & $R^{2}$ change \\
Level 2 & & $0.17(0.00)^{*}$ \\
Evaluations of quality of life & QOLS & $0.39(0.00)^{*}$ \\
& SF36-PCS & $-0.23(0.01)^{*}$ \\
& & $R^{2}$ change \\
Total $R^{2} 0.6 \mathrm{I}, F=21.83,(P<0.000) *$ & $0.186(0.00)^{*}$
\end{tabular}

Notes: Standardized regression coefficients are reported (numbers in parentheses are the $P$-values). *Significance level of 0.05 . Matrices is a subtest of the Wechsler Adults Intelligence Scale 3rd edition. DASS-depression and DASS-anxiety are subscales of the Depression Anxiety and Stress Scale.

Abbreviations: COWAT, controlled oral word association test; DASS, Depression Anxiety and Stress Scale; MARS-MFS, Memory Awareness Rating Scale-Memory Function Scale; $\mathrm{MCl}$, mild cognitive impairment; MMSE, mini-mental status exam; QOLS, quality of life scale; SF36-PCS, SF-36v2 ${ }^{\mathrm{TM}}$ physical component score. network DSSI, memory function MARS-MFS, Matrices, Logical I, and COWAT are mediated by level 2 QOLS and SF36 PCS.

\section{Discussion}

This study provides empirical support for a hierarchical model of PWB in MCI that explains $61 \%$ of the variance as measured here. The hypotheses that level 1 clinical aspects of MCI, depression, and social support would be interrelated, and that those primary aspects would influence secondary evaluations of life quality, were also supported. Further, the postulate that tertiary-level PWB would be significantly influenced by primary MCI and depression, as well as secondary evaluations of QoL, was supported. Moreover, our hypothesis that evaluations of QoL would mediate the effect of lower-level 1 variables on PWB was partially supported. Results obtained here are hence consistent with findings from studies of older adults that suggest that lower QoL and well-being are associated with higher memory concern, ${ }^{29,45}$ cognitive deficits, ${ }^{20}$ and negative affect. ${ }^{46}$ However, unlike those studies, analyses here examined clinical aspects of MCI, negative affect, and QoL within the one cohort, thus testing a more comprehensive model in a clinically relevant sample.

Our examination of clinical aspects of MCI indicated that memory concern in the form of complaints and self-rated memory function was significantly associated with cognitive and daily function, independently of negative affect. Memory concern was also significantly linked to a low satisfaction with social support and greater depressive symptoms. With the exception of cognitive and daily functions, which were not associated, all other level 1 factors were significantly linked together. These findings therefore provide some support for Jonker's conceptualization ${ }^{17}$ of primary disease and nondisease factors relevant to PWB.

Our findings also align with research that memory concerns are indeed reported by individuals with objective evidence of cognitive decline. ${ }^{47,48}$ Many studies have not reported significant associations between memory concern and cognitive function, finding rather that memory concern is related to psychopathology, personality traits such as neuroticism, and negative cognitive bias. ${ }^{29}$ In our study, however, the association between memory concern and cognition was independent of the links between memory complaint and self-rated memory function, and negative affect.

Lower cognitive performance in our MCI sample was associated with higher levels of negative affect, consistent with previous MCI research. ${ }^{49}$ Individuals with depression were excluded from this study and self-reported symptom 
levels were exceedingly low; nonetheless, mixed associations between cognition and negative affect were evident depending upon the scale. This highlights a previously known issue: that different depression scales can provide conflicting results. ${ }^{50}$ Moreover, the nature of any causal links between cognitive function and depression is controversial. In healthy adults, some research has suggested that perceived deterioration in memory may lead to anxiety, possibly fear about developing dementia, with depression as a natural response. ${ }^{51}$ Hence, poor cognitive function may lead to depression. Other research indicates a concurrent incidence between $\mathrm{MCI}$ and depressive symptoms. Therefore, another possibility is that they are comorbid conditions and the presence of hippocampal atrophy in both cognitive impairment and depression suggests a common biological process as well. Yet another alternative is that depression is a significant risk factor for subsequent cognitive deterioration. ${ }^{49,52}$

At this time, there is no consensus in this area. However, given that negative affect was here independently associated with cognitive function, social support, memory concern, and PWB, understanding and identifying negative affect in MCI represents a valuable potential treatment target.

Level 1 memory concern, cognitive function, capacity to complete daily activities, social support, and negative affect were significantly related to level 2 evaluations of QoL. Specifically, results here indicate that satisfaction with social support has a large and significant positive association with QoL, consistent with previous research. ${ }^{25}$ The association between memory concern and QoL is controversial because of the role of depression and negative cognitive bias or "affective distortion". ${ }^{46}$ In contrast, negative affect, specifically anxiety and depression, in this study was significantly linked to lower evaluations of QoL; a finding entirely consistent with previous research. For example, a review of Health Related Quality of Life (HR-QoL) in dementia indicated that decreased QoL was consistently associated with depression. ${ }^{53}$ Similarly, evaluations of QoL have been found to deteriorate with depression in clinical settings. ${ }^{54}$ Thus, findings here suggest complex interrelationships similar to those reported in a previous study of community dwelling elderly, which found that increasing severity of memory concern was associated with multiple factors including poor social network, negative age stereotyping, and depression. ${ }^{55}$

Within this study, cognitive function was also related to QoL. In mild dementia, lower level of cognition is linked to lower health related QoL. ${ }^{56}$ However, different cognitive functions had different relationships with each QoL outcome. A review examining the influence of specific cognitive func- tions on HR-QoL in neurological disease similarly identified differential impacts. ${ }^{57} \mathrm{~A}$ total of $92 \%$ of participants in this study were identified as having nonamnestic MCI. The cognitive deficits and risk profiles associated with nonamnestic $\mathrm{MCI}$ are heterogeneous, giving rise to the subtyping of MCI. ${ }^{58}$ Therefore, it is plausible that the various cognitive deficits in this cohort are differentially associated with QoL and further research is required.

Finally, analysis of level 3 PWB indicated that primary level clinical aspects of MCI and depression, and level 2 evaluations of QoL, were both predictive of PWB. Subjective memory concern was directly linked to PWB after controlling for depression. This result is consistent with several previous studies, ${ }^{29,45}$ however, conflicting results have also been noted. ${ }^{59}$ Results here suggest that subjective level of complaint and self-ratings of memory function have differential impact upon PWB. Inconclusive and equivocal findings regarding QoL and memory concern may therefore, in part, reflect different assessment approaches for subjective memory concern ${ }^{60}$ and QoL. ${ }^{5}$ Recommendations have been made to examine the subdivisions of subjective cognitive complaint, ${ }^{21}$ and this is supported here. Consequently, two practical clinical conclusions to draw from this study are, first, that measuring self-rated memory function in daily life may be more useful in understanding PWB than focusing on the presence or absence of complaint, and, second, that improving a person's memory function through external or internal aids may significantly improve PWB, potentially due to an increased sense of self efficacy.

The lack of consensus regarding the etiology of memory concern, ${ }^{61}$ and its association with psychopathology, has reinforced the notion that concern is purely subjective. As a result, individuals with complaints may be dismissed by health professionals as the "worried well". ${ }^{2}$ However, as findings here suggest, concern may be linked to subtle cognitive difficulties and reduced daily function, and it is possible that adults perceive such changes when traditional cognitive measures are insensitive. ${ }^{63}$ Furthermore, results from this study suggest that memory concerns in MCI are linked to lower ratings of QoL and PWB. In healthy adults, perceived deterioration in memory has been found to lead to anxiety. ${ }^{51}$ Here, anxiety was linked to reduced psychological well-being. Consequently, this evidence supports a general recommendation that memory concerns should not be underestimated in clinical settings. ${ }^{64}$ Clinicians' responses could focus on adjusting expectations, psychoeducation to alleviate anxiety, and practical strategies to minimize the impact of cognitive deficits. 
Our results also indicate that early subtle cognitive deficits are directly and significantly associated with PWB after controlling for depression. These results are consistent with findings from a systematic review that identified that even a mild deterioration in cognition has significant psychological impact. However, unlike previous research, results here also suggest that the negative impact of mild depressive and anxiety symptoms on PWB are mediated by evaluations of life quality across multiple domains. This finding may also have clinical implication. By improving aspects of a person's life, such as introducing increasing social support, recreational pursuits, and supporting community access, it is possible that the deleterious impact of depression and anxiety on PWB may be mitigated.

Previous research indicates that frailty and cognitive deficits determine QoL in MCI and early stage dementia. Therefore, not surprisingly, lower cognitive function was significantly associated with compromised physical-QoL measured on the SF36 PCS in this study. Contrary to expectation in the multivariate model, high SF36 PCS was related to a significant reduction in psychological well-being, but mediated the impact of memory concern, cognitive difficulties, and negative affect on psychological well-being.

This study has a number of limitations due to methodological constraints. The cross-sectional nature of the study restricts the extent to which causal inferences can be made. The sample was small, comprising only $100 \mathrm{MCI}$ individuals with subtle cognitive deficits (mean MMSE 27.4) and who were motivated enough to volunteer for the study, and, thus, their wider representativeness is not clear. Therefore, given this MCI profile, another possible limitation that remains is the evolving nature of MCI diagnosis. ${ }^{65}$ The impact of more severe memory deficits associated with amnestic-MCI was also not examined, and so results here may underestimate the burden of disability and reduced PWB in this group. In addition, being a convenience sample with psychopathology excluded, the associations between clinical depression and all factors, particularly PWB, could not be examined. Lastly, the sample completed a high level of education, and, within this range, lower education was significantly related to lower mood. Therefore, results may not necessarily apply to less educated individuals.

Individuals with $\mathrm{MCI}$ encounter various unique practical and emotional difficulties. In this study, we formally tested a hierarchical model in which clinical aspects of disease influence QoL, which in turn influences PWB. We found that clinical aspects of MCI were significantly associated with reduced PWB, whilst high QoL was associated with high PWB. Results here indicate that, for individuals with the subtlest of cognitive changes, their PWB may be at risk. Intervention targeting emotional, functional, and social factors, in addition to cognitive health, may optimize PWB outcomes, and, ideally, such treatment should commence when individuals first present with concerns for their memory function.

\section{Acknowledgments}

This study is part of the multicenter longitudinal SMART trial funded by a National Health and Medical Research Council (NHMRC) of Australia Dementia Research Grant, project grant ID No 512672 from 2008-2011. Additional funding for a research assistant position was sourced from the NHMRC Program Grant ID No. 568969, and the trial was supported by the University of Sydney and the University of New South Wales. MV was supported by a NHMRC Clinical Career Development Fellowship (1004156). We wish to acknowledge the contributions of Nalin Singh, Bernhard T Baune, and Henry Brodaty who were involved in the funding application, design, and general supervision of the SMART trial. In addition we acknowledge the roles of Michael Baker, Nidhi Jain, Nasim Foroughi, Jacinda Meiklejohn, and Guy Wilson in trial administration, recruitment, data collection, and data entry. Lastly, we are indebted to the participants who devoted their time to this project.

\section{Disclosure}

The authors report no conflicts of interest in this work.

\section{References}

1. Schölzel-Dorenbos CJ, van der Steen MJ, Engels LK, Olde Rikkert MG. Assessment of quality of life as outcome in dementia and MCI intervention trials: A systematic review. Alzheimer Dis Assoc Disord. 2007;21(2):172-178.

2. Brown J, Bowling A, Flyn T. Models of QoL: A Taxonomy, Overview and Systematic Review of the Literature, European Forum on Population Ageing Research. 2004

3. St John P, Montgomery PR. Cognitive impairment and life satisfaction in older adults. Int J Geriatr Psychiatry. 2010;25:814-821.

4. Billotta C, Bowling A, Nicolini P, et al. Older people's quality of life of life (OPQOL) scores and adverse health outcomes at one-year follow-up. A prospecive cohort study study on older outpatients living in the communty in Italy. Health Qual Life Outcomes. 2011;9:72.

5. Halvorsrud L, Kalfoss M. The conceptualization and measurement of quality of life in older adults: A review of empirical studies published during 1994-2006. Eur J Ageing. 2007;4(4):229-246.

6. Cummins RA. On the trial of the gold standard for subjective wellbeing. Soc Indic Res. 1995;43:307-334.

7. Depp C, Jeste DV. Definitions and Predictors of Successful Aging: A Comprehensive Review of Larger Quantitative Studies. Am J Geriatr Psychiatry. 2006;14(1):6-20.

8. Depp CA, Glatt SJ, Jeste DV. Recent advances in research on successful or healthy aging. Curr Psychiatry Rep. 2007;9:7-13. 
9. Lehnert K, Sudeck G, Conzelmann A. Subjective well-being and exercsie in the second half of life: a critical review of the theoretical approaches. Eur Rev Aging Phys Act. 2012;9:87-102.

10. Keyes CL, Shmotkin D, Ryff CD. Optimizing well-being: The empirical encounter of two traditions. J Pers Soc Psychol. 2002;82(6): 1007-1022

11. Arent SM, Landers DM, Etnier JL. The effects of exercise on mood in older adults: A meta-analytic review. J Aging Phys Act. 2000;8: 407-430.

12. Andrews FM, Withey SB. Social Indicators of Well-Being: America's Perception of Life Quality. New York: Plenum Press; 1976.

13. Ryff CD, Keyes CL. The structure of Psychological Well-Being revisited. J Pers Soc Psychol. 1995;69(4):719-727.

14. Netz Y, Wu MJ, Becker BJ, Tenebaum G. Physical activity and psychological well-being in advanced age: A meta-analysis of intervention studies. Psychol Aging. 2005;20(2):272-284.

15. Lawton MP. Assessing quality of life in Alzheimer disease research. Alzheimer Dis Assoc Disord. 1997;11 Suppl 6:91-99.

16. Ryan RM, Deci EL. On happiness and human potentials: A review of research on hedonic and eudaimonic well-being. Annu Rev Psychol. 2001;52:141-166.

17. Jonker C, Gerritsen DL, Bosboom PR, Van Der Steen JT. A model for quality of life measures in patients with dementia: Lawton's next step. Dement Geriatr Cogn Disord. 2004;18(2):159-164.

18. Ring L, Höfer S, McGee H, Hickey A, O’Boyle CA. Individual quality of life: Can it be accounted for by psychological or subjective wellbeing? Soc Indic Res. 2007;82(3):443-461.

19. Dear K, Henderson S, Korten A. Well-being in Australia. Findings from the national survey of mental health and well-being. Soc Psychiatry Psychiatr Epidemiol. 2002;37:507-509.

20. Mhaoláin AM, Gallagher D, Crosby L, et al. Frailty and quality of life for people with Alzheimer's disease and mild cognitive impairment. Am J Alzheimers Dis Other Demen. 2012;27(1):48-54.

21. Mark RE, Sitskoorn MM. Are subjective cognitive complaints relevant in preclinical Alzheimer's disease? A review and guidelines for healthcare professionals. Rev Clin Gerontol. 2013;23(1):61-74.

22. Winbald B, Palmer K, Kivipelto M, et al. Mild Cognitive impairmentbeyond controversies, towards consensus: report of the International Working Group on Mild Cognitive impairment. J Intern Med. 2004;256: 240-246.

23. Huckans M, Hutson L, Twamley E, Jak A, Kaye J, Storzbach D. Efficacy of cognitive rehabilitation therapies for mild cognitive impairment (MCI) in older adults: Working toward a theoretical model and evidence-based interventions. Neuropsychol Rev. 2013;(23): 63-80.

24. Banerjee S, Smith SC, Lamping DL, et al. Quality of life in dementia : more than just cognition. An analysis of associations with quality of life in dementia. J Neurol Neurosurg Psychiatry. 2008;77:146-148.

25. Bowling A, Hankins M, Windle G, Bilotta C, Grant R. A short measure of quality of life in older age: the performance of the brief Older Person's Quality of Life Questionnaire (OPQOL-Brief). Arch Gerontol Geriatr. 2013;56:181-187.

26. Livingston G, Cooper C, Woods J, Milne A, Katona C. Successful ageing in adversity: The LASER-AD longitudinal study. J Neurol Neurosurg Psychiatry. 2008;79(6):641-645.

27. Cummins RA. Fostering quality of life. InPsych The Bulletin of the Australain Psychological Society. 2013;35(1):8-11.

28. Gates N, Valenzuela M, Sachdev PS, et al. Study of Mental Activity and Regular Training (SMART) in at risk individuals: A randomised double blind, sham controlled, longitudinal trial. BMC Geriatr. 2011;11:19.

29. Montejo P, Montenegro M, Fernández MA, Maestú F. Memory complaints in the elderly: Quality of life and daily living activities. A population based study. Arch Gerontol Geriatr. 2012;54:298-304.

30. Clare L, Wilson BA, Carter G, Roth I, Hodges JR. Assessing awareness in early-stage Alzheimer's disease: Development and piloting of the memory awareness rating scale. Neuropsychol Rehabil. 2002;12(4):341-362.
31. Folstein MF, Folstein SE, McHugh PR. 'Mini-Mental State': A practical method for grading the cognitive state of patients for the clinician. J Psychiatr Res. 1975;12:189-198.

32. Rosen WG, Mohs RC, Davis KL. A new rating scale for Alzheimer's disease. Am J Psychiatry. 1984;141(11):1356-1364.

33. Lezack M, Howieson DB, Loring DW, editors. Neuropsychological Assessment. 4th ed. Oxford University Press; 2004.

34. Wechsler D. Wechsler Memory Scale 3rd Edition. Harcourt Brace and Company; 1997.

35. Sivan AB. Benton Visual Retention Test 5th Edition. Harcourt Brace and Company; 1992.

36. Wechsler D. Wechsler Adult Intelligence Scales 3rd Edition. Harcourt Brace and Company; 1997.

37. Hindmarch I, Lehfeld H, de Jongh P, Erzigkeit H. The Bayer Activities of Daily Living Scale (B-ADL). Dement Geriatr Cogn Disord. 1998; 9 Suppl 2:20-26.

38. Brink TL, Yesavage JA, Lum O, Heersema P, Adey M, Rose TL. Screening tests for geriatric depression. Clin Gerontol. 1982;1:37-43.

39. Spreen O, Strauss E, editors. A Compendium of Neuropsychological Tests. Administration, Norms, and Commentary. 2nd ed. Oxford University Press; 1998

40. Lovibond SH, Lovibond PF. Manual for the Depression Anxiety and Stress Scales (DASS) 1993. Psychology Foundation Monograph, (University of New South Wales NSW 2052, Australia).

41. Koenig HG, Westlund RE, George LK, Hughes DC, Blazer DG, Hybels C. Abbreviating the Duke Social Support Index for use in chronically ill elderly individuals. Psychosomatics. 1993;34:61-69.

42. Burkhardt CS, Anderson KL. The Quality of Life Scale (QOLS): reliability, validity and utilization. Health Qual Life Outcomes. 2003;1:60.

43. Ware JE, Sherbourne CE. The MOS 36-item short form health survey (SF-36). I. Conceptual framework and item selection. Med Care. 1992;30(6):473-483.

44. Baron RM, Kenny DA. The moderator-mediator variable distinction in social psychological research: Conceptual, strategic, and statistical considerations. J Pers Soc Psychol. 1986;51:1173-1182.

45. Trigg R, Watts S, Jones R, Tod A. Predictors of quality of life ratings from persons with dementia: the role of insight. Int J Geriatr Psychiatry. 2011;26:83-91.

46. Berwig M, Leicht H, Hartwig K, Gertz HJ. Self-rated quality of life in mild cognitive impairment and Alzheimer's disease: The problem of affective distortion. GeroPsych (Bern). 2011;24(1):45-51.

47. Mitchell AJ. The clinical significance of subjective memory complaints in the diagnosis of mild cognitive impairment and dementia. Int $J$ Geriatric Psychiatry. 2008;23:1191-1202.

48. Jessen F, Wiese B, Cvetanovska G, et al. Patterns of subjective memory impairment in the elderly: Association with memory performance. Psychol Med. 2007;37(12):1753-1762.

49. Rosenberg PB, Mielke MM, Appleby BS, Oh ES, Geda YE, Lyketsos CG. The association of neuropsychiatric symptoms in MCI with incident dementia and Alzheimer disease. Am J Geriatr Psychiatry. 2013;21:685-695.

50. Defrancesco M, Marksteiner J, Deisenhammer EA, Hinterhuber H, Weiss EM. [Association of Mild Cognitive Impairment (MCI) and depression]. Neuropsychiatr. 2009;23(3):144-150. German.

51. Verhaeghen P, Geraerts N, Marcoen A. Memory complaints, coping, and well-being in old age: A systemic approach. Gerontologist. 2000;40(5):540-548.

52. van den Kommer TN, Comijs HC, AArtsen MJ, Huisman M, Deeg DJH, Beekman ATF. Depression and cognition: how do they interrelate in old age? Am J Geriatr Psychiatry. 2013;21:398-410.

53. Banerjee S, Samsi K, Petrie CD, et al. What do we know about quality of life in dementia? A review of emerging evidence on the predictive and explanatory value of disease specific measures of health related quality of life in people with dementia. Int J Geriatr Psychiatry. 2009;24:15-24.

54. Netuveli G, Blane D. Quality of life in older ages. Br Med Bull. 2008;85:113-126. 
55. Derouesné C, Alperovitch A, Arvay N, et al. Memory complaints in the elderly: A study of 367 community-dwelling individuals from 50 to 80 years old. Arch Gerontol Geriatr Suppl. 1989;1:151-163.

56. Hurt CS, Banerjee S, Tunnard C, et al. Insight, cognition and quality of life in Alzheimer's disease. J Neurol Neurosurg Psychiatry. 2010;81(3):331-336.

57. Mitchell AJ, Kemp S, Benito-León J, Reuber M. The influence of cognitive impairment on health-related quality of life in neurological disease. Acta Neuropsychiatr. 2010;22:2-13.

58. Sachdev PS, Lipnicki DM, Crawford J, et al. Risk profiles of subtypes of mild cogitive impairment: the sydney memory and ageing study. J Am Geriatr Soc. 2012;60:24-33.

59. Mol M, Carpay M, Ramakers I, Rozendaal N, Verhey F, Jolles J. The effect of perceived forgetfulness on quality of life in older adults; a qualitative review. Int J Geriatr Psychiatry. 2007;22:393-400.

60. Abdulrab K, Heun R. Subjective Memory Impairment. A review of its definitions indicates the need for a comprehensive set of standardised and validated criteria. Eur Psychiatry. 2008;23:321-330.
61. Dux MC, Woodard JL, Calamari JE, et al. The moderating role of negative affect on objective verbal memory performance and subjective memory complaints in healthy older adults. $J$ Int Neuropsychol Soc. 2008;14(2):327-336.

62. Alladi S, Arnold R, Mitchell J, Nestor PJ, Hodges JR. Mild cognitive impairment: applicability of research criteria in a memory clinic and characterization of cognitive profile. Psychol Med. 2006;36(4): 507-515.

63. Geerlings MI, Jonker C, Bouter LM, Adèr HJ, Schmand B. Association between memory complaints and incident Alzheimer's disease in elderly people with normal baseline cognition. Am J Psychiatry. 1999; 156(4):531-537.

64. Jonker C, Geerlings MI, Schmand B. Are memory complaints predictive for dementia? A review of clinical and population-based studies. Int $J$ Geriatr Psychiatry. 2000;15:983-991.

65. Han JW, Kima TH, Leeb SB, et al. Predictive validity and diagnostic stability of mild cognitive impairment subtypes. Alzheimers Dement. 2012;8:553-559. 


\section{Supplementary material}

Table SI Regression of memory complaint and subjective memory function measures on cognitive and daily function variables, and daily function on cognitive variables controlling for demographic and negative affect variables

\begin{tabular}{llll}
\hline $\begin{array}{llll}\text { Independent } \\
\text { variables }\end{array}$ & \multicolumn{3}{l}{ Dependent variables } \\
\cline { 2 - 4 } & MCS & MARS-MFS & B-ADL ${ }^{\#}$ \\
\hline $\begin{array}{l}\text { Cognitive function } \\
\text { ADAS learning }\end{array}$ & $0.21(0.13)$ & $0.09(0.40)$ & $0.12(0.27)$ \\
ADAS-Cog & $0.21(0.010)^{*}$ & $-0.32(0.00)^{*}$ & $-0.02(0.83)$ \\
MMSE & $0.03(0.97)$ & $0.05(0.56)$ & $-0.04(0.69)$ \\
TMT A & $-0.12(0.22)$ & $0.10(0.34)$ & $-0.03(0.72)$ \\
TMT B & $-0.16(0.13)$ & $0.15(0.14)$ & $0.02(0.80)$ \\
Matrices & $-0.03(0.7 I)$ & $-0.02(0.83)$ & $0.09(0.39)$ \\
Similarities & $-0.21(0.03)^{*}$ & $0.03(0.76)$ & $-0.00(0.97)$ \\
Animal fluency & $-0.13(0.20)$ & $0.18(0.09)$ & $-0.05(0.61)$ \\
COWAT & $-0.05(0.61)$ & $0.14(0.20)$ & $-0.10(0.34)$ \\
Logical I & $-0.24(0.00)^{*}$ & $0.15(0.12)$ & $-0.09(0.37)$ \\
Logical II & $-0.22(0.00)^{*}$ & $0.24(0.01)^{*}$ & $-0.11(0.26)$ \\
BVRT & $0.00(0.96)$ & $0.18(0.08)$ & $0.02(0.84)$ \\
SDMT & $-0.16(0.11)$ & $0.17(0.10)$ & $-0.04(0.69)$ \\
Daily function & & & \\
B-ADL ${ }^{\#}$ & $0.29(0.00)^{*}$ & $-0.35(0.00)^{*}$ & - \\
\hline
\end{tabular}

Notes: The regression coefficients shown for daily function and cognitive function variables were obtained from separate regression analyses, one for each of these variables, with demographic (age, education, and sex) and negative affect variables included in the equations as control variables. Standardized regression coefficients are reported, with $P$-values in parentheses; *Significance level of 0.05 . \#High score indicates greater difficulty. Matrices and Similarities are subtests of the Wechsler Adult Intelligence Scale 3rd edition. Logical I immediate and Logical II delayed are logical memory subscales of Wechsler Memory Scale 3rd edition. Animal fluency refers to category word fluency.

Abbreviations: ADAS-Cog, Alzheimer's Disease Assessment Scale-cognitive; ADAS Learning, list learning trials from ADAS-Cog; B-ADL, Bayer-Activities of Daily Life; BVRT, Benton Visual Retention Test revised 5th edition; COWAT, controlled oral word association test; MARS-MFS, Memory Awareness Rating Scale-Memory Functioning Scale; MCS, memory concern scale; MMSE, mini-mental state exam; SDMT, Symbol Digit Modality Test; TMT, Trail Making Test; TMT A, numbers; TMT $B$, numbers and letters. 
Table S2 Regression analyses level I clinical aspects of $\mathrm{MCl}$ and social support and negative affect

\begin{tabular}{|c|c|c|c|c|c|}
\hline \multirow{2}{*}{$\begin{array}{l}\text { Clinical aspects of mild cognitive } \\
\text { impairment as independent variables }\end{array}$} & \multicolumn{5}{|c|}{ Dependent variables } \\
\hline & DSS & GDS & DASS anxiety & DASS depression & DASS stress \\
\hline \multicolumn{6}{|l|}{ Covariates } \\
\hline Age & $-0.02(0.83)$ & $-0.17(0.08)$ & $0.06(0.53)$ & $-0.01(0.85)$ & $-0.10(0.30)$ \\
\hline Sex & $0.05 I(0.62)$ & $0.07(0.43)$ & $-0.14(0.15)$ & $-0.10(0.30)$ & $-0.00(0.94)$ \\
\hline Education & $0.078(0.45)$ & $-0.11(0.25)$ & $-0.27(0.00)^{*}$ & $-0.22(0.02)^{*}$ & $-0.13(0.20)$ \\
\hline \multicolumn{6}{|l|}{ Memory concern } \\
\hline MCS & $0.04(0.66)$ & $0.29(0.00)^{*}$ & $0.03(0.72)$ & $0.13(0.18)$ & $0.08(0.40)$ \\
\hline MARS-MFS & $0.21(0.04)^{*}$ & $-0.29(0.00)^{*}$ & $-0.15(0.13)$ & $-0.25(0.01)^{*}$ & $-0.25(0.01)^{*}$ \\
\hline \multicolumn{6}{|l|}{ Daily function } \\
\hline B-ADL & $-0.14(0.16)$ & $0.18(0.06)$ & $0.09(0.06)$ & $0.14(0.15)$ & $0.16(0.10)$ \\
\hline \multicolumn{6}{|l|}{ Cognitive function } \\
\hline ADAS-learning & $0.12(0.29)$ & $-0.14(0.18)$ & $-0.06(0.55)$ & $-0.15(0.17)$ & $-0.07(0.5 \mathrm{I})$ \\
\hline ADAS-Cog & $0.26(0.02)^{*}$ & $0.28(0.00)^{*}$ & $0.19(0.07)$ & $0.25(0.01)$ & $0.34(0.00)^{*}$ \\
\hline MMSE & $0.10(0.33)$ & $-0.11(0.27)$ & $0.00(0.96)$ & $-0.01(0.85)$ & $-0.07(0.48)$ \\
\hline TMT A & $0.19(0.07)$ & $0.19(0.07)$ & $0.32(0.00)^{*}$ & $0.33(0.00)^{*}$ & $0.21(0.04)^{*}$ \\
\hline TMT B & $0.05(0.620)$ & $0.20(0.06)$ & $0.16(0.11)$ & $0.17(0.10)$ & $0.11(0.28)$ \\
\hline Matrices & $0.06(0.58)$ & $0.02(0.81)$ & $-0.04(0.66)$ & $0.00(0.95)$ & $-0.13(0.21)$ \\
\hline Similarities & $0.02(0.85)$ & $-0.10(0.36)$ & $-0.12(0.27)$ & $-0.05(0.6 \mathrm{I})$ & $-0.05(0.62)$ \\
\hline Animal fluency & $0.15(0.19)$ & $0.02(0.85)$ & $-0.09(0.38)$ & $-0.12(0.26)$ & $0.00(0.94)$ \\
\hline COWAT & $0.23(0.04)^{*}$ & $-0.09(0.43)$ & $-0.29(0.00)^{*}$ & $-0.36(0.00)^{*}$ & $0.09(0.40)$ \\
\hline Logical I & $0.07(0.49)$ & $-0.20(0.05)$ & $-0.11(0.26)$ & $-0.03(0.73)$ & $0.07(0.48)$ \\
\hline Logical II & $0.10(0.33)$ & $-0.22(0.02)^{*}$ & $-0.12(0.22)$ & $-0.05(0.60)$ & $0.01(0.85)$ \\
\hline BVRT & $0.25(0.02)$ & $-0.16(0.14)$ & $-0.24(0.02)^{*}$ & $-0.28(0.00)^{*}$ & $-0.35(0.00)^{*}$ \\
\hline SDMT & $0.05(0.59)$ & $-0.17(0.11)$ & $-0.22(0.03)^{*}$ & $-0.28(0.00)^{*}$ & $-0.12(0.26)$ \\
\hline
\end{tabular}

Notes: The regression coefficients shown for daily function and cognitive function variables were obtained from separate regression analyses, one for each of these variables, with age, sex, and education included in the equations as control variables. The regression coefficients for age, sex, and education are those for analyses when all of these variables were entered simultaneously in the equations. Standardized regression coefficients are reported, numbers in parentheses ( ) are the $P$-values, and $*$ indicates significance level of 0.05 . Matrices and Similarities are subtests of the Wechsler Adult Intelligence Scale 3rd edition. Animal fluency refers to category word fluency. Logical I and Logical II are logical memory subscales of Wechsler Memory Scale 3rd edition.

Abbreviations: ADAS-Cog, Alzheimer's Disease Assessment Scale-cognitive; ADAS-learning, list learning trial of ADASC-Cog; B-ADL, Bayer-Activities of Daily Life; BVRT, Benton Visual Retention Test revised 5th edition; COWAT, controlled oral word association test; DASS, Depression Anxiety and Stress Scale; DSS, Duke Social Support; GDS, Geriatric Depression Scale; MARS-MFS, Memory Awareness Rating Scale-Memory Functioning Scale; $\mathrm{MCl}$, mild cognitive impairment; MCS, memory concern scale; MMSE, mini-mental state exam; SDMT, Symbol Digit Modality Test; TMT, Trail Making Test; TMT A, numbers; TMT B, numbers and letters. 
Table S3 Regression analyses for the effects of level I variables on level 2 evaluations of quality of life variables

\begin{tabular}{|c|c|c|c|c|}
\hline \multirow{3}{*}{$\begin{array}{l}\text { Level I independent } \\
\text { variables }\end{array}$} & \multicolumn{2}{|c|}{ Dependent variables } & \multicolumn{2}{|c|}{ Level 2 evaluations of quality of life } \\
\hline & \multicolumn{2}{|l|}{ QOLS } & \multicolumn{2}{|l|}{ SF36 PCS } \\
\hline & All variables & Reduced model & All variables & Reduced model \\
\hline \multicolumn{5}{|l|}{ Demographics } \\
\hline Age & $0.09(0.33)$ & $0.21(0.02)^{*}$ & $-0.35(0.00)^{*}$ & \\
\hline Sex & $0.10(0.28)$ & & $0.14(0.12)$ & \\
\hline Education & $0.05(0.59)$ & & $0.00(0.96)$ & \\
\hline \multicolumn{5}{|l|}{ Negative affect } \\
\hline GDS & $0.32(0.00)^{*}$ & & $0.13(0.15)$ & \\
\hline DASS anxiety & $-0.29(0.00)^{*}$ & & $-0.21(0.02)^{*}$ & $-0.27(0.01)^{*}$ \\
\hline DASS depression & $-0.43(0.00)^{*}$ & $-0.19(0.09)^{*}$ & $-0.20(0.03)^{*}$ & $-0.24(0.04)^{*}$ \\
\hline DASS stress & $-0.29(0.00)^{*}$ & & $0.00(0.09)$ & $0.36(0.00)^{*}$ \\
\hline \multicolumn{5}{|l|}{ Social support } \\
\hline DSS & $0.43(0.00)^{*}$ & $0.41(0.00)^{*}$ & $-0.02(0.8 \mathrm{I})$ & \\
\hline \multicolumn{5}{|l|}{ Memory concern } \\
\hline MCS & $0.08(0.4 I)$ & & $-0.02(0.78)$ & $0.26(0.01)^{*}$ \\
\hline MARS-MFS & $0.27(0.00)^{*}$ & $0.17(0.04)^{*}$ & $0.15(0.13)$ & \\
\hline \multicolumn{5}{|l|}{ Daily function } \\
\hline B-ADL & $0.06(0.55)$ & $-0.17(0.05)^{*}$ & $0.08(0.32)$ & $-0.24(0.04)^{*}$ \\
\hline \multicolumn{5}{|l|}{ Cognitive function } \\
\hline ADAS learning & $0.18(0.09)$ & $0.20(0.03)^{*}$ & $0.26(0.0 \mathrm{II}) *$ & $0.44(0.00)^{*}$ \\
\hline ADAS-Cog & $0.24(0.02)^{*}$ & & $0.15(0.134)$ & \\
\hline MMSE & $-0.05(0.59)$ & & $0.08(0.39)$ & \\
\hline TMT A & $-0.25(0.01)^{*}$ & & $0.09(0.38)$ & \\
\hline TMT B & $-0.24(0.02)^{*}$ & $-0.19(0.04)^{*}$ & $0.25(0.01)^{*}$ & $-0.27(0.00)^{*}$ \\
\hline Matrices & $-0.06(0.55)$ & & $0.12(0.21)$ & $0.29(0.00)^{*}$ \\
\hline Similarities & $-0.10(0.36)$ & $-0.18(0.04)^{*}$ & $0.16(0.11)$ & \\
\hline Animal fluency & $-0.00(0.93)$ & $-0.18(90.05)^{*}$ & $-0.01(0.86)$ & $-0.25(0.00)^{*}$ \\
\hline COWAT & $0.13(0.25)$ & & $0.18(0.08)$ & $-0.23(0.05)^{*}$ \\
\hline Logical I & $-0.15(0.13)$ & $-0.20(0.02)^{*}$ & $0.10(0.30)$ & \\
\hline Logical II & $-0.04(0.66)$ & & $0.16(0.09)$ & \\
\hline BVRT & $0.22(0.04)^{*}$ & & $0.03(0.73)$ & \\
\hline SDMT & $0.16(0.14)$ & & $0.31(0.00)^{*}$ & \\
\hline$R^{2}$ & $0.52(0.000)^{*}$ & $0.48(0.000)^{*}$ & $0.5 \mathrm{I}(0.000)^{*}$ & $0.45(0.000)^{*}$ \\
\hline
\end{tabular}

Notes: Standardized regression coefficients are reported. Numbers in parentheses are the $P$-values. *Significance level of 0.05 . Matrices and similarities are subtests of the Wechsler Adult Intelligence Scale 3rd edition. Animals refers to category word fluency. Logical I and logical II are Logical memory subscales of Wechsler Memory Scale 3rd edition.

Abbreviations: ADAS-Cog, Alzheimer's Disease Assessment Scale-cognitive; ADAS learning, list learning trial of ADASC-Cog; B-ADL, Bayer-Activities of Daily Life; BVRT, Benton Visual Retention Test revised 5th edition; COWAT, controlled oral word association test; DASS, Depression Anxiety and Stress Scale; DSS, Duke Social Support; GDS, Geriatric Depression Scale; MARS-MFS, Memory Awareness Rating Scale-Memory Functioning Scale; MCS, memory concern scale; MMSE, mini-mental state exam; QOLS, quality of life scale; SDMT, Symbol Digit Modality Test; SF36 PCS, physical component score; TMT, Trail Making Test; TMT A, numbers; TMT B, numbers and letters.

\section{Publish your work in this journal}

Clinical Interventions in Aging is an international, peer-reviewed journal focusing on evidence-based reports on the value or lack thereof of treatments intended to prevent or delay the onset of maladaptive correlates of aging in human beings. This journal is indexed on PubMed Central, MedLine, the American Chemical Society's 'Chemical Abstracts Ser- vice' (CAS), Scopus and the Elsevier Bibliographic databases. The manuscript management system is completely online and includes a very quick and fair peer-review system, which is all easy to use. Visit http://www.dovepress.com/testimonials.php to read real quotes from published authors. 